\title{
POPQ system and dynamic MRI in assessment of female genital prolapse*
}

\author{
Mohamed N. El-Gharib ${ }^{1 \#}$, Manal A. Farahat ${ }^{1}$, Mahmoud Daoud ${ }^{2}$ \\ ${ }^{1}$ Department of Obstetrics \& Gynaecology, Faculty of Medicine, Tanta University, Tanta, Egypt \\ ${ }^{2}$ Department of Radiodiagnosis, Faculty of Medicine, Tanta University, Tanta, Egypt \\ Email: " mohgharib@hotmail.com
}

Received 26 January 2013; revised 27 February 2013; accepted 8 March 2013

\begin{abstract}
Aim: To assess the role of Magnetic resonance imaging (MRI) and pelvic organ prolapse quantification (POPQ) system in the evaluation of female pelvic organ prolapse. Design: Prospective study. Setting: Tanta University Hospital. Patients: The study was carried out on 60 patients having clinical manifestations suggesting pelvic floor weakness. Intervention: All the patients were subjected to history taking, physical examination including POPQ, and pelvic MRI (static and dynamic) examination. Outcome Measures: Quantitative measurements of genital prolapse. Results: All patients showed loss of the normal position of the perineal plate indicating generalized weakness of the whole pelvic floor muscles. Dynamic MRI exhibited pelvic floor abnormalities in $\mathbf{4 6}$ patients who did not show any abnormalities on the static images. Conclusion: Dynamic MRI is a helpful tool in defining the nature and quantitative measurements of pelvic organ prolapse. Also, MRI has and advantage over POPQ system in diagnosing lateral prolapse.
\end{abstract}

Keywords: MRI; Pelvic Floor; Cystocele; Cystorectocele; Uterine Prolapse; Enterocele

\section{INTRODUCTION}

MRI is the diagnostic imaging modality of choice for many disorders of the female pelvis. Benign uterine disease is well-demonstrated using rapid sequences and minimal examination time, whereas evaluation of malignancy is best performed with high-resolution techniques. Wide-spread availability of ultrafast sequences has expanded MR imaging applications to include evaluation of pelvic floor relaxation and fetal anomalies [1].

Disadvantages of MRI are its high cost and long scanning time. Its excellent tissue contrast underscores

\footnotetext{
*The authors have no declaration of interests to report.

${ }^{\#}$ Corresponding author.
}

its importance in the evaluation of adnexal masses, however, because it allows specific diagnoses of fat, blood, and fibrous tissue. Even if normal in size, an ovary may present with tiny foci of endometrial implants or dermoid cysts that are only identifiable on MR imaging; however, MR imaging is generally used as a problem-solving modality. When ultrasound results are inconclusive, the use of MR imaging may alter treatment decisions, eliminate the need for surgery, and result in reduced overall costs [1].

The use of MRI for pelvic organ prolapse was introduced by Yang et al. in 1991 [2]. It providing standard fluoroscopic information on organ prolapse, MR can be used to evaluate for subtle superior rectovaginal septal defects, uterine and pelvic floor abnormalities, and lateral prolapse. The MRI study is also less invasive than is fluoroscopy, because bladder catheterization is not necessary and there is no use of ionizing radiation. The main drawback of MRI is that the patient is usually supine for the examination [3].

The aim of the work was to assess the role of MRI and POPQ system in the evaluation of female pelvic organ prolapse.

\section{PATIENTS}

This study included 60 patients having clinical manifestations of pelvic organ prolapse ( $\geq$ Stage I), otherwise were considered normal.

\section{METHODS}

All the patients were subjected to: history taking, clinical examination including assessment of pelvic organ prolapse according to POPQ system [4], routine laboratory investigations, liver and renal function, and pelvic MRI examination. The patients were examined with use of 1.5 Tesla MR. The patients were positioned supine without tilting of the pelvis to either side. Pads were put under neath the buttocks to encourage the patients to strain forcefully. The patients may also flex their knees in order to simulate a sitting position, and hence strain more 
easily and forcefully. Next we combined static and dynamic (functional) pulse sequences: Axial and sagittal slices, respectively, were obtained by means of a T2weighted turbo spin-echo sequence. Dynamic studies were performed with balanced fast field echo (BFFE) at rest and after maximal straining (Valsalva maneuver). The standard slice orientation included mid-sagittal and axial planes. The axial images provided information about the urogenital hiatus ballooning and its contents if any. During the measurements the patient was asked to relax the pelvic floor muscles then asked to increase the intra- abdominal pressure by straining and then relax again. Weakness of pelvic floor muscles can be described by measuring the pelvic organ descent below the pubococcygeal line (PCL), the perineal plate angle on the midsagittal images and the urogenital hiatus width on the axial images.

\section{RESULTS}

The mean age of the patients was $38 \pm 4.2$ years, mean parity $4.2 \pm 1.4$, MBI $27.6 \pm 3.5$, urinary symptoms in $81.67 \%$.

The main complaints were: sensation of heaviness or pulling in the pelvis with something protruding from the vagina in $34 / 60$ cases, low back pain (24/60), sexual dysfunction, including decreased libido (36/60), and difficulty achieving orgasm (24), constipation (12/60), urinary incontinence (18/60).

Stage of prolapse at baseline according to POPQ system, Stage one 13 cases $(21.7 \%)$, Stage two 18 cases $(30.0 \%)$, Stage three 29 cases $(48.3 \%)$.

Clinical examination revealed that 24 women had anterior and posterior prolapse, 30 had anterior prolapse only, and 6 had uterine and apical prolapse. The MRI findings showed that among 30 women diagnosed clinically as anterior prolapse, three women had lateral cystocele. Meanwhile, The POPQ evaluation did not find the lateral cystocele to be related to the prolapse score.

In addition, MRI illustrated that in 26 patients the urinary bladder was just touching the pubococcygeal ligament (PCL) and caudal inclination of the perineal plate at straining sequences, 18 patients showed descent of the urinary bladder and the uterine cervix below the PCL, the last 13 patients showed anterior descent of the urinary bladder and 3 cases exhibited lateral descent of the urinary bladder. The uterine cervix and lower segment of the uterus were below the PCL, associated with enterocele and caudal inclination of the levator plate on straining sequences. All patients showed loss of the normal position of the perineal plate indicating generalized weakness of the whole pelvic floor muscles (Figures 1 and 2).

Only half of the cases exhibited abnormalities on the static MRI images i.e. none of the organs was seen touching or below the PCL. Dynamic MRI revealed
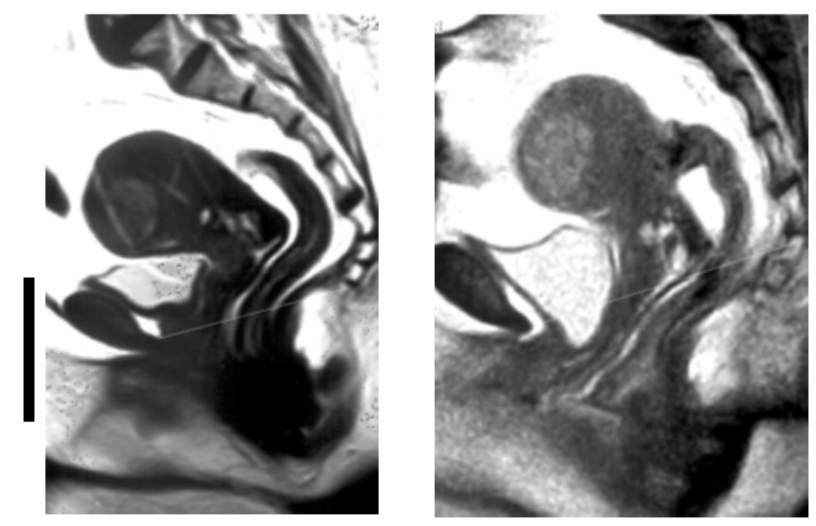

Figure 1. A 30-year-old multiparous patient clinically diagnosed as stress incontinence during rest (left) and during straining (right).
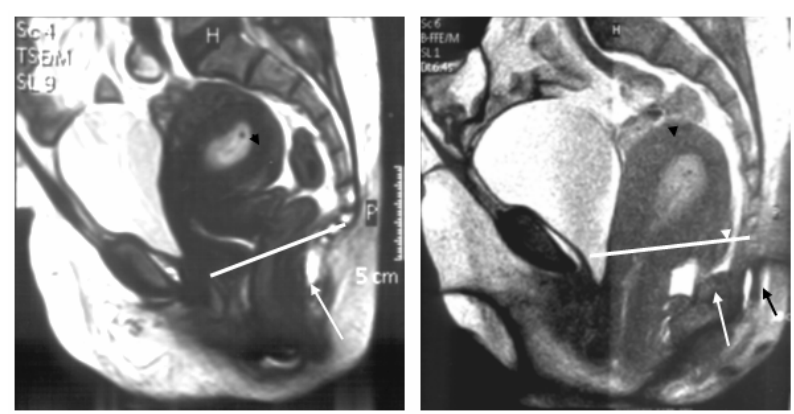

Figure 2. A 32-year-old multiparous patient clinically diagnosed as uterine prolapse. Mid-sagittal images at rest (left) and straining (right), showing an RVF uterus (black arrow head) as well as rectal descent (white arrow) and marked caudal inclination of the perineal plate (black arrow). At straining showing evidence of two enterocele (white arrow heads) as well as marked generalized pelvic floor descent with all pelvic organs (urinary bladder, cervix and rectum) below the PCL (white line).

pelvic organ prolapse in double the cases found by static MRI as depicted in Tables $\mathbf{1}$ and $\mathbf{2}$.

\section{DISCUSSION}

Pelvic prolapse is a common problem affecting women of all ages. Pelvic prolapse involves the herniation of various portions of the vaginal wall. Symptoms vary according to the area of the vagina affected. Proper evaluation is imperative for providing proper treatment. Various surgical approaches to repair have been developed and techniques continue to evolve [5].

Pelvic floor dysfunction encompasses a variety of fascial and anatomic defects that can include a combination of cystocele, rectocele, uterine prolapse, enterocele and vault prolapse. Accurate diagnosis of the coexisting abnormalities is essential in planning reconstructive procedures so that the risks of recurrence and reoperation can be minimized. At this time, dynamic magnetic resonance imaging is the study of choice to evaluate 
Table 1. MRI findings in cases with pelvic floor abnormalities.

\begin{tabular}{ccccccc}
\hline Pathology & \multirow{2}{*}{$\begin{array}{c}\text { Number } \\
\text { of patients }\end{array}$} & $\%$ & \multicolumn{3}{c}{ MR findings } \\
\cline { 4 - 6 } & & \multicolumn{2}{c}{$\begin{array}{c}\text { Static Dynamic } \\
\text { MRI }\end{array}$} & MRI & Other findings \\
\hline Anterior prolapse & 27 & 45 & - & 30 & $\begin{array}{c}\text { Lateral prolapse } \\
\text { in 3 cases }\end{array}$ \\
$\begin{array}{c}\text { Anterior and } \\
\text { posterior prolapse } \\
\begin{array}{c}\text { Apical and uterine } \\
\text { prolapse }\end{array}\end{array}$ & 24 & 40 & 8 & 16 & $\begin{array}{c}\text { Fibroid } \\
\text { seen in 3 cases } \\
\text { RVF uterus }\end{array}$ \\
\hline
\end{tabular}

Table 2. POP measurements during straining.

\begin{tabular}{ccc}
\hline Measurement & Range & Mean \\
\hline Aa & -3 to +3 & $-0.72 \pm 1.9$ \\
Ba & -3 to +7 & $-0.50 \pm 2.4$ \\
Ap & -3 to +3 & $-1.36 \pm 1.7$ \\
Bp & -3 to +7 & $-1.4 \pm 2.2$ \\
C & -14 to -7 & $-4.7 \pm 4.6$ \\
D & -15 to +8.5 & $-7.0 \pm 3.6$ \\
TVL & 4.5 to 13 & $8.5 \pm 1.7$ \\
GH & 1 to 11 & $4.3 \pm 1.5$ \\
PB & 0.5 to 7.5 & $3.4 \pm 1.2$ \\
\hline
\end{tabular}

the female pelvis and delineate the possible components of pelvic floor dysfunction [6].

Fielding [7] stated that approximately $50 \%$ of parous women have pelvic floor relaxation and $20 \%$ of that group is symptomatic enough to seek treatment. Symptoms include urinary and fecal incontinence and protrusion of tissue, usually the cervix or uterus, through the pelvic floor. Most women with urinary incontinence can be diagnosed.

We based our study by using the PCL as a reference point for measuring pelvic organ descent in relation to it as it is a best reference line in cases of cystocele and uterine prolapse. We measured the distance of the bladder-neck, distal edge of cervix/posterior fornix, and the most ventrocaudal point of the ventral rectal wall, respectively, to the pubococcygeal line (PCL), the horizontal tangent of the inferior rim of the pubic bone, and the line drawn through the long axis of the pubic bone. On functional MRI the best correlation with the clinical results was achieved using the PCL for the anterior compartment. This agree with the findings of Lienemann et al. [8].

In the current study we found that there was great association between parity status and the high incidence of pelvic floor dysfunction as patients of pelvic floor dysfunction in our study were found in patients with more than two normal vaginal deliveries, however $71.4 \%$ of our patients were complaining of symptoms related to pelvic floor dysfunction representing.

Hoyte et al. [9] stated that sagittal midline images using an ultrafast T2-weighted pulse sequence with the woman at rest and at maximal strain quantify the descent of all three compartments at once and can be used to identify enterocele, sigmoidocele, and anterior urethral rotation and kinking. Descent of organs $2 \mathrm{~cm}$ below the pubococcygeal line usually indicates the need for surgery in a symptomatic patient, thin, axial, high-resolution T2weighted images detail muscle atrophy and tears. Lateral deviation of the vagina usually indicates a paravaginal fascial tear. 4 cases in our study showed descent of pelvic organs (UB in one case and uterus combined with cystocele in three cases) $2 \mathrm{~cm}$ below the pubococcygeal line, and they were symptomatic and they underwent surgical correction.

Pannu [3] found that sagittal images similar to fluoroscopy are obtained to diagnose prolapse, fluoroscopic criteria also have been applied to MR. This is most commonly for the diagnosis of rectocele and enterocele, in all cases, abnormalities on MR have to be correlated with patient history and clinical findings.

Gufler et al. concluded that dynamic MRI of the pelvic floor with a half-Fourier RARE sequence can reliably detect descents of all three pelvic compartments, that it requires no contrast agent, and that no radiation exposure is involved [10].

The advantages of dynamic magnetic resonance imaging include excellent depiction of the soft tissues and pelvic organs, and their fluid content during various degrees of pelvic strain. To our knowledge whether it is cost-effective in this manner has not been determined [11].

Signs of genital prolapse are frequently found in the female general population but are seldom symptomatic. Of factors associated with genital prolapse found in this study, pelvic floor muscle strength appears to be the only one that could be affected [12].

In the present study we found that 26 patients showed that the urinary bladder just touching the PCL and caudal inclination of the perineal plate at straining sequences, 18 patients showed descent of the urinary bladder and the uterine cervix below the PCL, 16 patients showed also descent of the urinary bladder, uterine cervix and lower segment of the uterus below the PCL, associated with enterocele and caudal inclination of the levator plate on straining sequences, All patients showed loss of the normal position of the perineal plate indicating generalized weakness of the whole pelvic floor muscles.

The results of the current investigation supported the importance of the dynamic pulse sequences in the diagnosis of genital descent. We observed pelvic floor ab- 
normalities in 46 patients which did not show any abnormalities on the static images.

Lastly, we concluded that Dynamic MRI is a helpful tool in defining the nature, degree and quantitative measurements of genital prolapse because physical examination is often insufficient in defining the nature and degree of visceral prolapse. Moreover, quantitative measurements are difficult to obtain by clinical examination only.

\section{REFERENCES}

[1] Schwartz, L.B., Panageas, E. Lange, R. Rizzo, J., Comite, F. and McCarthy, S. (1995) Female pelvis: Impact of MR imaging on treatment decisions and net cost analysis. $\mathrm{Ob}$ stetrical \& Gynecological Survey, 50, 36-38. doi:10.1097/00006254-199501000-00020

[2] Yang, A., Mostwin, J.L., Rosenshein, N.B. and Zerhouni, E.A. (1991) Dynamic evaluation of pelvic floor descent in women using fastscan MRI and cinematic display. $R a-$ diology, 179, 25-33.

[3] Pannu, H.K. (2003) Dynamic MR imaging of female organ prolapse. Radiologic Clinics of North America, 41, 409-423. doi:10.1016/S0033-8389(02)00120-3

[4] Bump, R.C., Mattiasson, A., Bo, K., Brubaker, L.P., Delancey, J.O., Klarskov, P., Shull, B.L. and Smith, A.R. (1996) The standardization of terminology of female pelvic organ prolapse and pelvic floor dysfunction. American Journal of Obstetrics \& Gynecology, 175, 10-17. doi:10.1016/S0002-9378(96)70243-0

[5] Kobashi, K.C. and Leach, G.E. (2000) Pelvic prolapse. The Journal of Urology, 164, 1879-1890. doi:10.1016/S0022-5347(05)66909-1
[6] Larissa, V.R. and Shlomo, R. (2001) Diagnostic imaging of pelvic floor dysfunction. Current Opinion in Urology, 11, 423-428. doi:10.1097/00042307-200107000-00015

[7] Fielding, J.R. (2003) MR imaging of the female pelvis. Radiologic Clinics of North America, 41, 179-192. doi:10.1016/S0033-8389(02)00063-5

[8] Lienemann, A., Sprenger, D., Janßen, U., Grosch, E., Pellengahr, C. and Anthuber, C. (2004) Assessment of pelvic organ descent by use of functional cine-MRI: Which reference line should be used? Neurourology and Urodynamics, 23, 33-37. doi:10.1002/nau.10170

[9] Hoyte, L., Schierlitz, L., Zou, K., Flesh, G. and Fielding, J.R. (2001) Two- and 3-dimensional MRI comparison of levator ani structure, volume, and integrity in women with stress incontinence and prolapse. American Journal of Obstetrics \& Gynecology, 185, 11-19. doi: $10.1067 / \mathrm{mob} .2001 .116365$

[10] Gufler, H., Laubenberger, J., De Gregorio, G., Dohnicht, S. and Langer, M. (1999) Pelvic floor descent: Dynamic MR imaging using a half-fourier RARE sequence. Journal of Magnetic Resonance Imaging, 9, 378-383. doi:10.1002/(SICI)1522-2586(199903)9:3<378::AID-JM RI3>3.0.CO;2-9

[11] Marinkovic, S.P. and Stanton, S.L. (2004) Incontinence and voiding difficulties associated with prolapse. The Journal of Urology, 171, 1021-1028. doi:10.1097/01.ju.0000111782.37383.e2

[12] Eva, C.S., Arne, V.F.T., Gosta, T. and Kurt, F.S. (1999) Signs of genital prolapse in a Swedish population of women 20 to 59 years of age and possible related factors. American Journal of Obstetrics \& Gynecology, 180, 299. 305. doi:10.1016/S0002-9378(99)70203-6 